\title{
Clinical Outcome of Chronic Leg and Foot Ulcers Treated with Autologous Platelet Rich Fibrin
}

\author{
Neethu Rasajnam ${ }^{1}$, Sreejith Kalathummarathu², Shehadad Kammili³, Sreedevi Menon ${ }^{4}$ \\ 1,3,4 Department of Physical Medicine \& Rehabilitation, Govt. Medical College, Kozhikode, Kerala, India. \\ ${ }^{2}$ Department of Physical Medicine \& Rehabilitation, Govt. Medical College, Kottayam, Kerala, India.
}

\section{ABSTRACT}

\section{BACKGROUND}

Chronic leg and foot ulcers are a major health problem and leads to negative physical, social and psychological impact on patients and families. A high percentage of chronic ulcers do not adequately heal or quickly relapse with conventional treatment. They need advanced treatment modalities. Emerging cellular therapies such as platelet-rich concentrate therapy is an advanced modality. Platelet rich fibrin (PRF) is a second-generation platelet concentrate, consists of fibrin three-dimensional polymerized matrix, with the incorporation of platelets and leukocytes. The objective of the study was to assess clinical outcome of chronic leg and foot ulcers treated with autologous platelet rich fibrin.

\section{METHODS}

This prospective study included 30 patients with chronic leg or foot ulcers of various aetiologies, 19 patients had diabetic foot ulcers, 7 venous ulcers, 1 trophic ulcer, 2 pressure ulcers and 1 traumatic ulcer. Autologous PRF treatment outcome was measured by percentage of improvement in area and volume of the ulcer. We also studied other ulcer characteristics including exudate type, pus culture and sensitivity status, presence of undermining, type of tissue in ulcer bed and comorbidities.

\section{RESULTS}

From the 30 ulcers treated with PRF, the mean percentage of improvement in area and volume of ulcers were $98.3 \%$ and $98.8 \%$ respectively at the end of 2 months. $80 \%$ ulcers healed completely. There was also reduction of pain and exudate after first week of treatment. Other than increased callus formation in some ulcers, no other adverse events were reported following PRF treatment.

\section{CONCLUSIONS}

PRF is a safe, easy-to-use, cost-effective treatment method with significant potential for healing chronic ulcers.

\section{KEY WORDS}

PRF-Platelet Rich Fibrin
Corresponding Author: Dr. Shehadad Kammili, Kammili (H), Atholi (PO), Kozhikode - 673315,

Kerala, India.

E-mail:drshehadad@gmail.com

DOI: $10.14260 /$ jemds $/ 2022 / 7$

How to Cite This Article:

Rasajnam N, Kalathummarathu S, Kammili $S$, et al. Clinical outcome of chronic leg and foot ulcers treated with autologous platelet rich fibrin. J Evolution Med Dent Sci 2022;11(01):33-38, DOI: 10.14260/jemds/2022/7

Submission 09-12-2021, Peer Review 16-12-2021, Acceptance 08-01-2022, Published 13-01-2022.

Copyright (C) 2022 Neethu Rasajnam et al. This is an open access article distributed under Creative Commons Attribution License [Attribution 4.0 International (CC $B Y 4.0)]$ 


\section{BACKGROUND}

Chronic ulcers are major health problem worldwide. They impose a significant burden to personal, professional and social level. Chronic ulcers are defined as wounds which have failed to proceed through an orderly and timely reparative process to produce anatomic and functional integrity. ${ }^{1}$ The normal wound healing involves the following phases: rapid haemostasis, inflammation, mesenchymal cell proliferation, angiogenesis, re-epithelialization, proper synthesis, crosslinking, and alignment of collagen to provide strength to the healing tissue. These phases must occur in the proper sequence, at a specific time, and continue for a specific duration at an optimal intensity. ${ }^{2}$ In chronic ulcers, these orderly arrangements of phases are interrupted because of various intrinsic or extrinsic factors.

There are various aetiologies for causing chronic leg and foot ulcers. It includes venous, arterial, diabetic, pressure, traumatic ulcers. Even in developed countries 1-2 \% of population is likely to have a chronic wound during their life time. $^{3}$ Conventional treatment includes wound cleaning, necrotic tissue debridement, moist dressing, local application of ointments, colloid dressing, avoiding risk factors and mechanical off-loading. Advanced treatments include skin grafting, vacuum assisted closure, cellular therapy, hyperbaric oxygen therapy and surgical management. ${ }^{4}$

Cellular therapy is a major breakthrough treating chronic leg and foot ulcers. Autologous platelet rich fibrin is a newer treatment modality. It is now increasingly being used in various medical fields. PRF is a second-generation platelet concentrate, first described by Choukroun et al. ${ }^{5} \mathrm{PRF}$ is a fibrin matrix consist of platelet concentrate and leucocyte cytokines. Platelet and cytokines in the PRF promote healing and has supportive effect on immune system. Fibrin matrix act as supporting system and causes slow polymerization. Platelet rich fibrin accelerates wound healing by releasing various growth factors like TGF, VEGF, EGF, FGF, CTGF. Each growth factor is a signalling molecule responsible for a specific activity in the wound healing cascade. ${ }^{5}$

Autologous platelet rich fibrin is made from patient's own blood by method of centrifugation. It includes collection of whole venous blood in each test tube (amount $5 \mathrm{ml}$ in each) under aseptic precautions without any anticoagulants. Then immediately centrifuged at $3000 \mathrm{rpm}$ for $15 \mathrm{~min}$. This results in the formation of three fractions: upper straw-coloured acellular plasma (platelet-poor plasma); middle layerplatelet rich fibrin; lower- red blood cells (RBCs)). PPP was aspirated out and the PRF gel was separated from underlying RBC layer by the use of sterile stainless-steel blade. Preparation of PRF is simplified and cost-effective process. It has favourable healing due to slow polymerization, helps in haemostasis and more efficient cell migration, proliferation than platelet rich plasma. It is also used for tendon surgeries and bone graft surgeries and in implant dentistry. ${ }^{5}$

There are not much studies from Kerala showing effectiveness of PRF in chronic leg and foot ulcer healing. Hence, we choose this subject as our research topic. In this study, we wanted to assess clinical outcome of local application of autologous platelet rich fibrin for ulcer management. The variables like wound length, width, depth, area, volume, healing time of ulcer were assessed before and after PRF therapy.

\section{Objectives of the Study}

1. To assess clinical outcome of chronic leg and foot ulcers treated with autologous platelet rich fibrin.

2. To assess change in area and volume of chronic leg and foot ulcers treated with autologous platelet rich fibrin.

\section{METHODS}

This prospective study was conducted from February 2018 to February 2019 in the Department of Physical Medicine and Rehabilitation, Government Medical College, Kozhikode.

\section{Study Population}

Patients with chronic leg and foot ulcer attending Department of Physical Medicine and Rehabilitation treated by either conventional methods or by application of platelet rich fibrin. This study was done by following up the group of patients to whom platelet rich fibrin dressing was given and whose age was more than 18 years. Informed written consent was obtained from the subjects prior to the study.

\section{Inclusion Criteria}

1. Chronic leg and foot ulcers more than 6 weeks duration.

2. Age more than 18 years with informed consent.

3. Both males and females.

4. Baseline ulcer area between $3-20 \mathrm{~cm}^{2}$

\section{Exclusion Criteria}

1. Persons with uncontrolled diabetes mellitus with HbA1C more than 10

2. Ulcer with osteomyelitis

3. Patients taking anticoagulant/ antithrombotic/ immunosuppressive drugs

4. Poorly nourished and debilitated patients

5. Patient with history of bleeding disorder

Persons with chronic leg or foot ulcer who were posted for autologous platelet rich fibrin therapy and who met the inclusion criteria were enrolled into the study. Informed written consent was obtained prior to the study. The PRF was prepared by centrifuging $10 \mathrm{ml}$ of patient's blood at $3000 \mathrm{rpm}$ speed for 15 minutes. At the end of $15 \mathrm{~min}$ there are 3 layers-upper layers of straw-coloured acellular plasma, lower layer of RBC and middle layer of fibrin clot. The middle layer of fibrin clot is separated carefully under aseptic precautions and is dressed on the ulcer using sterile dressing pad. The procedure is repeated at weekly intervals.

All participants underwent detailed history taking and local examination of ulcer prior to PRF therapy. The following parameters were evaluated during examination: type, site, depth, width, length, area and volume of ulcer, presence of exudate and type of tissue present in the wound bed. Using these parameters, the ulcers were assessed every week while they were on topical autologous PRF therapy till 2 months or until the ulcer healed completely. 
Other characteristics like age, gender, occupation and comorbidities were also entered into a previously prepared proforma.

\section{Measurement Documentation}

- The length, width and depth of the ulcer were measured using a thread and a scale. This was done before the treatment, before repeating the treatment each time at weekly intervals and after the treatment was completed (the final measurement).

- Digital photographs were taken before starting the treatment, before repeating the treatment each time at weekly intervals and after the treatment was completed.

- Ulcer area calculated by using formula for an ellipse: length $\mathrm{x}$ width $\mathrm{x} 0.7854$ (an ellipse is closer to an ulcer area than a square or rectangle that would be described by simple length $\mathrm{x}$ width)

- Volume of ulcer calculated by formula (length $\mathrm{x}$ width $\mathrm{x}$ 0.7854 ) $\mathrm{x}$ depth.

\section{Statistical Analysis}

All the data were coded and entered in Microsoft Excel sheet, rechecked and analysed using Statistical Package for Social Science (SPSS version 20) software. Quantitative variable presented as mean and standard deviation. We performed paired $t$ test to compare the baseline and post PRF area and volume of ulcers in the study group. $P$ value less than 0.05 is taken as significant.

\section{Ethical Clearance}

The study protocol was submitted to Institutional Research Committee as well as the Institutional Ethics Committee of the Government Medical College, Kozhikode and clearance was obtained for conducting the study. Ethical clearance was obtained from the Institutional Human Ethics Committee.

- Informed consent was obtained from all the study participants.

- All the information that was collected was kept confidential.

- No procedure was carried out which directly or indirectly produced risk to the subjects.

\section{RESULTS}

This study was performed on 30 patients with chronic leg or foot ulcers of various aetiologies. The results were analysed as follows.

\section{Demographic Profile of Patients \\ Age}

The age of the patients ranged from 48 to 74 years and mean \pm SD was $60.3 \pm 10.15$ years.

\section{Gender Distribution}

In the study there were 19 (63\%) male patients and 11 (36\%) female patients.
Ulcer Characteristics

Aetiology of Ulcers

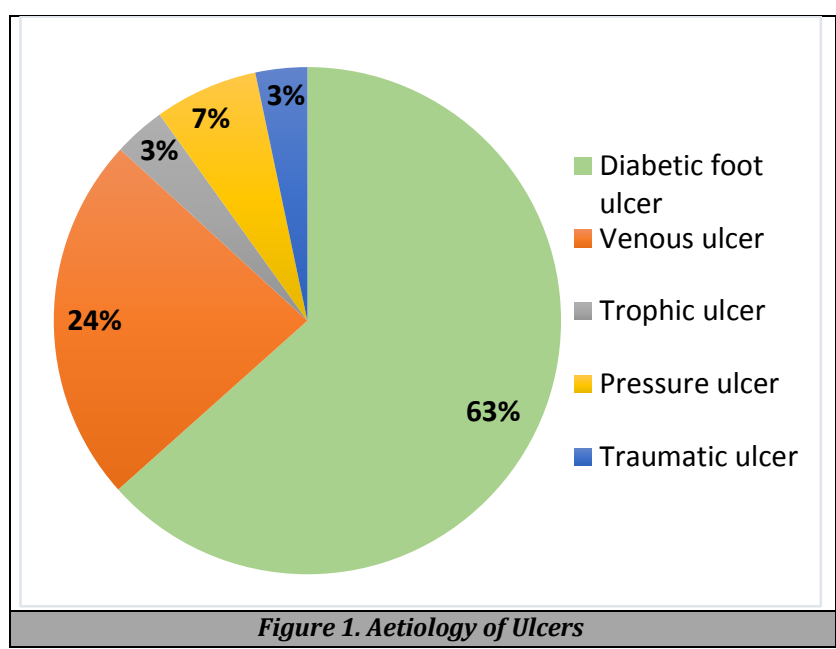

In our study we had 19 (63.4\%) diabetic foot ulcers, 7 (23.4\%) venous ulcers, 1 (3.3\%) trophic ulcer, 2 (6.6\%) pressure ulcers and $1(3.3 \%)$ traumatic ulcer.

\section{Site of Ulcer}

$70 \%$ of ulcers were on plantar aspects of foot, $23.4 \%$ of ulcers on gaiter's area, 3.3\% of ulcer on dorsum of foot and $3.3 \%$ ulcer on lateral aspect of ankle.

\section{Duration of Ulcer Prior to PRF Therapy}

The duration of ulcers prior to autologous platelet rich fibrin therapy ranged from 12 weeks to 44 weeks, with a mean \pm SD of $22.23 \pm 8.08$ weeks.

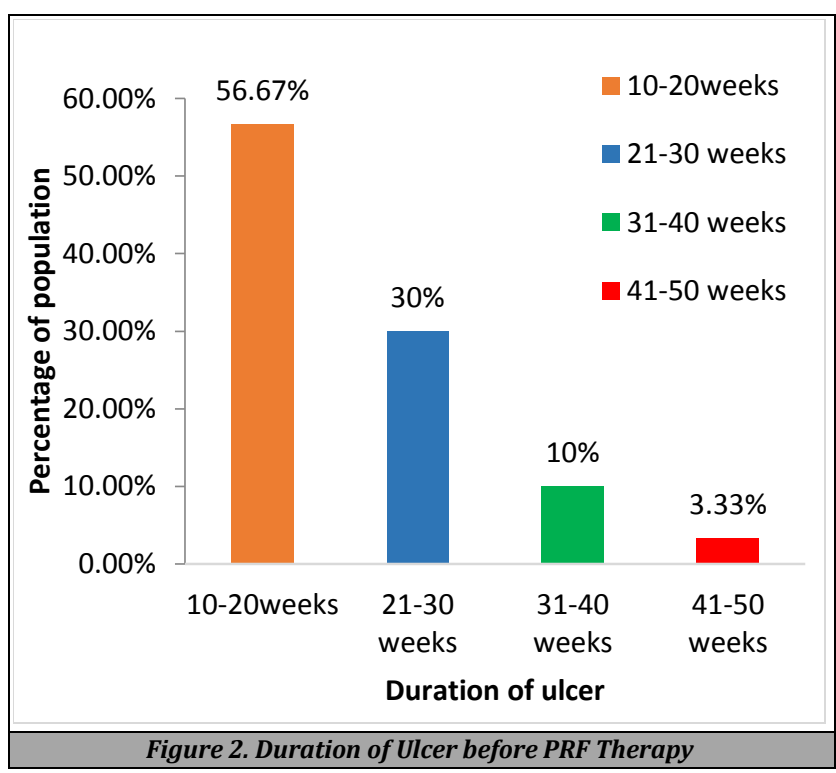

Area of the Ulcer

The baseline mean area of ulcers was $5.63 \mathrm{~cm}^{2}$ (SD 3.22). The final mean area at the end of maximum of 2 months was $0.15 \mathrm{~cm}^{2}$ (SD 0.38). P value was found to be 0.000 . Hence, the result is clinically significant. The mean percentage of improvement in area of ulcers was $98.3 \%$ (SD 3.4). 


\section{Percentage Improvement in Area of Ulcer at the End of Study}

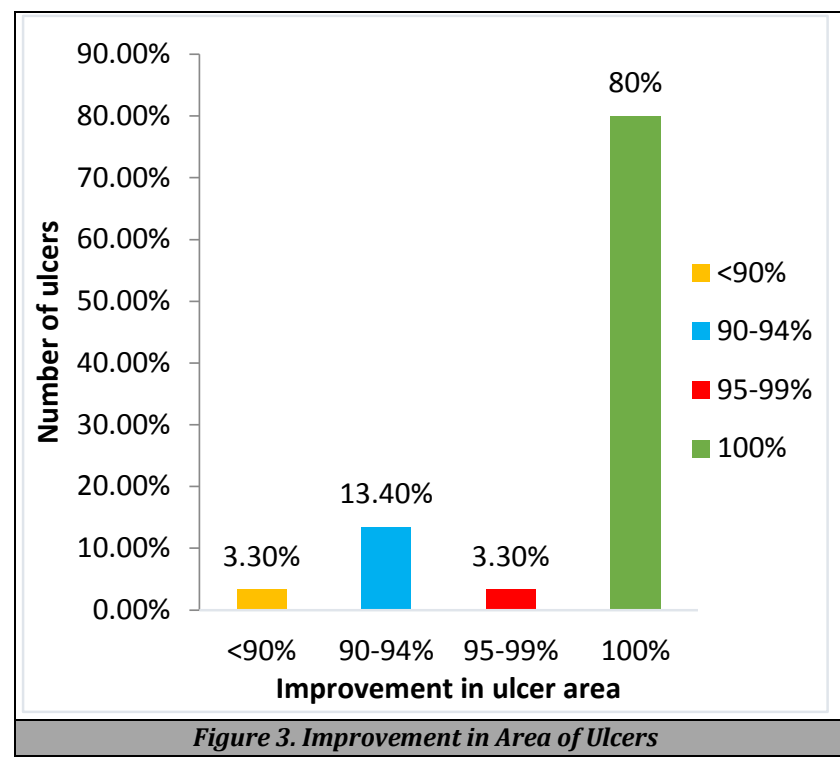

Of 30 ulcers included in the study, 24 ulcers showed 100 $\%$ improvement in area after PRP therapy.

\section{Volume of the Ulcers}

The baseline mean volume of ulcers was $6.16 \mathrm{~cm}^{3}$ (SD 5.25). The final mean volume at the end of maximum of 2 months was $0.11 \mathrm{~cm}^{3}$ (SD 0.31). P value was found to be 0.000 . Hence the result is clinically significant.

The mean percentage of improvement in volume of ulcers was $98.8 \%$ (SD 2.8).

\section{Percentage Improvement in Volume of Ulcer at the End of Study}

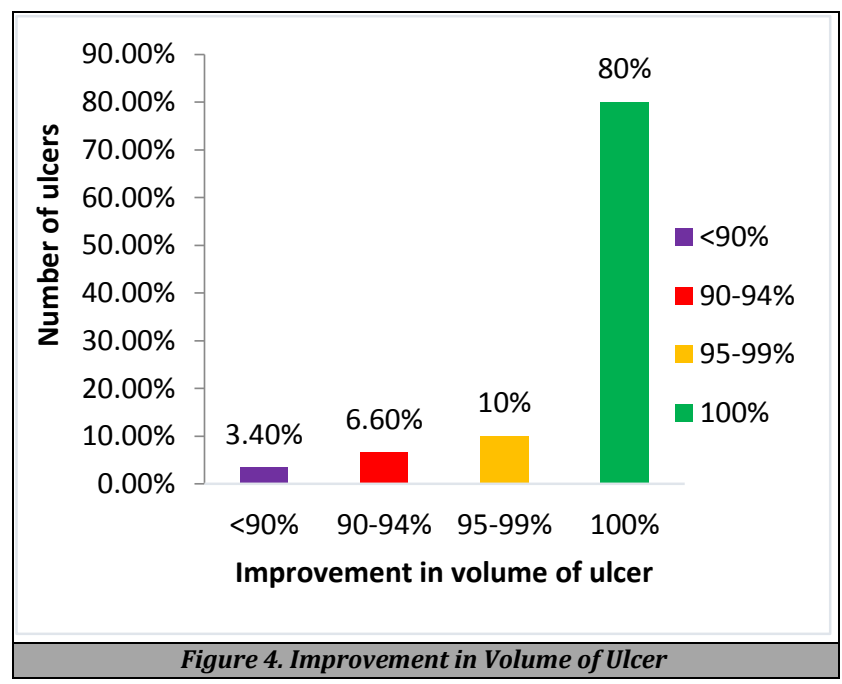

Of 30 ulcers treated with platelet rich fibrin, 24 (80\%) ulcers showed $100 \%$ improvement and one ulcer showed $88 \%$ improvement at the end of study period.

\section{Co-morbidities}

In this study population, $63.3 \%$ had diabetes mellitus, $60 \%$ had hypertension and $15 \%$ had dyslipidaemia.

\section{Treatment Undergone}

Number of PRF Applications

The mean number of PRF applications is 5 (SD 1.32), with range from 3 to 8 applications.

\section{Complete Closure of Ulcer}

For the analysis, complete closure is defined as complete epithelialization in the absence of exudate. Out of 30 ulcers treated, $24(80 \%)$ showed complete closure, $6(16 \%)$ ulcers showed $>90 \%$ closure and remaining 1 ulcer showed $>85 \%$ closure at the end of study period.

\section{Time to Closure}

Out of the 30 ulcers treated, 24 showed complete closure. In that group, the mean time to complete closure is 4.6 weeks (SD 1.0), with range from 3 to 7 weeks.

\section{Diabetic Ulcer}

In our study, there were 19 (63.4\%) diabetic foot ulcers. Of them, 13 showed complete closure at the end of study period.

\begin{tabular}{|c|c|c|c|c|c|c|c|}
\hline & \multirow[t]{2}{*}{$\begin{array}{l}\text { Duration of } \\
\text { Ulcer Prior } \\
\text { to PRF } \\
\text { (weeks) }\end{array}$} & \multicolumn{2}{|c|}{$\begin{array}{c}\text { Baseline Area \& } \\
\text { Volume }\left(\mathrm{cm}^{2}\right)\end{array}$} & \multicolumn{2}{|c|}{$\begin{array}{c}\text { Post PRF Area } \\
\text { \& Volume } \\
\left(\mathrm{cm}^{3}\right)\end{array}$} & \multicolumn{2}{|c|}{$\begin{array}{c}\text { \% of } \\
\text { Improvement } \\
\text { in Area \& } \\
\text { Volume }\end{array}$} \\
\hline & & Area & Volume & Area & Volume & Area & Volume \\
\hline Mean & 24.84 & & & 0.24 & 0.17 & 97.46 & 98.13 \\
\hline SD & 8.8 & 3.8 & 6.4 & 0.46 & 0.38 & 4.12 & 3.47 \\
\hline Minimum & 14 & 3.14 & 1.35 & 0 & 0 & 87 & 88 \\
\hline Maximum & 44 & 18.84 & 24.49 & 1.76 & 1.58 & 100 & 100 \\
\hline
\end{tabular}

The studied diabetic foot ulcers showed significant improvement in area and volume with PRF therapy ( $p$ value $<0.05$ ). The number of PRF applications needed ranged from 3 to 6 .

\section{Venous Ulcers}

This study included 7 venous ulcers. The duration of ulcers prior to PRF therapy ranged from 16 to 22 weeks with mean 18 weeks. The baseline mean area and volume of ulcers were 6.48 (SD 1.56) $\mathrm{cm}^{2}$ and 5.44 (SD 1.71) $\mathrm{cm}^{3}$ respectively. All of them showed complete closure at the end of study period. The number of PRF application required ranged from 5 to 6 . The mean duration for complete closure with PRF was 5.43 (SD 0.53) weeks.

\section{Pressure Ulcers}

This study included 2 pressure ulcers with mean baseline area and volume of $4.47 \mathrm{~cm}^{2}$ and $4.47 \mathrm{~cm}^{3}$ respectively. Both showed complete closure at the end of study with PRF therapy. The mean time required for complete closure was 4.5 weeks.

\section{Trophic and Traumatic Ulcers}

This study included 1 trophic and 1 traumatic ulcer. Both were fully healed with PRF therapy.

\section{DISCUSSION}

Chronic ulcers are a major cause of morbidity and mortality with increasing prevalence due to increase of risk factors. 
The various causes include venous dysfunction, diabetes mellitus, infections, peripheral neuropathy, pressure and atherosclerosis. Emerging cellular therapies such as plateletrich concentrate therapy has gathered considerable attention for its potential use in the field of regenerative medicine for ulcer management.

Platelet rich fibrin is a second-generation platelet rich concentrate, a simplified preparation without any biochemical blood handling. The beneficial effect of PRF in the healing of chronic leg ulcers is by its high concentration of platelets and leukocytes and its ability of long-term release of growth factors specific to fibrin matrix. ${ }^{5}$

In this study, total of 30 patients with ulcers were treated with PRF. Among the ulcers treated, there were $7(23.4 \%)$ venous ulcers, $19(63.4 \%)$ diabetic ulcers, $1(3.3 \%)$ trophic ulcer, 2(6.6\%) pressure ulcers and $1(3.3 \%)$ traumatic ulcer.

A study conducted by Dr. Gajjala et al. included 30 nonhealing ulcers of various aetiologies. The total mean percentage improvement in the area and volume of the ulcer with PRF observed were $95.8 \%$ and $100 \%$ respectively by $6^{\text {th }}$ sitting and all ulcers closed by a maximum of six PRF sitting. ${ }^{6}$ In our study the mean percentage of improvement in area and volume of ulcers were $98.3 \%$ and $98.8 \%$ respectively at the end of 2 months.

A randomized controlled trial conducted by Anirudh Somani et al. compared the efficacy of autologous plateletrich fibrin versus saline dressing in chronic venous leg ulcers and it showed that the ulcers treated with PRF had better and faster improvement when compared to saline dressing. ${ }^{7}$ The mean reduction in ulcer area was $82.51 \%$ for PRF and $42.74 \%$ for saline. There was complete closure of ulcer only in PRF dressings. In our study all venous ulcers completely healed after treatment with PRF.

A study carried out by Sean M. O Connell concluded that the treatment of venous and non-venous ulcers had different outcomes. He showed that the patients who had venous ulcers and were treated with platelet rich fibrin had a total closure of wound in $66.7 \%$ of patients. Whereas patients who had ulcers and were not of venous origin had total closure in only $44 \%$ of patients. ${ }^{8}$

Hence, he proved that PRF was very efficacious in the treatment of venous ulcers. In our study we had assessed 7 venous ulcers, all of them healed fully at the end of the study period. From the remaining 23 non-venous origin ulcers, $73 \%$ showed complete healing at the end of 2 months. Both these studies showed similar result.

Diabetic foot is a frequent complication of DM. Ulceration occurs as a result of trauma in the presence of neuropathy and/or peripheral vascular disease with infection as a secondary phenomenon following disruption of the protective epidermis. A study conducted by Nelson R Pinto et al. evaluated effect of PRF on various chronic leg ulcers, of which 10 cases were diabetic foot ulcers. All diabetic foot ulcers showed full closure within a 3-month period. ${ }^{9}$ In our study, there were 19 (63.4\%) diabetic foot ulcers. Of them, 13 showed complete closure at the end of study period. The mean percentage of improvement in the area and volume of the ulcers were 97.46 and 98.13 respectively.

Platelet-rich plasma (PRP) is a first-generation platelet concentrate. However, the short duration of growth factors and cytokine release, and its poor mechanical properties have resulted in search of new material. Platelet rich fibrin is a second-generation platelet concentrate, made by simplified processing without biochemical blood handling. It does not require the use of bovine thrombin or anticoagulants. PRF releases high quantities of growth factors for 7 days which make it different from PRP. The easily applied PRF acts like a fibrin bandage with biochemical components that have well known synergistic effects on healing processes. ${ }^{5}$

A study by Azariah Johnson Samuel Pravin et al. compared the effect of autologous platelet rich plasma versus leucocyte-platelet rich fibrin (L-PRF) in chronic non-healing leg ulcers. This study included 30 chronic ulcers. The mean duration of healing of the ulcers were 5.7 weeks in L-PRF group and 6.5 in PRP group and about $100 \%$ resolution was seen in 11 of the ulcers in L-PRF and 8 of the ulcers in PRP at the end of the 6 th treatment $\left(73.3 \%\right.$ vs. 53.3\%). ${ }^{10}$ They concluded that L-PRF is more efficacious and provides quicker healing that PRP and it was attributed to its antiinflammatory properties and protection against infections. In our study $100 \%$ healing was seen in $80 \%$ ulcers at the end of study period which is a good outcome, even though there is no comparison with any other agent like PRP.

PRF enhances wound healing by the release of growth factors. These growth factors stimulate mesenchymal cell recruitment, proliferation, extracellular matrix degeneration, and cell differentiation for tissue regeneration. This membrane protects open wounds and accelerates healing, favours the development of micro vascularization, and also guides epithelial cell migration to its surface. ${ }^{6}$

In our study there is significant reduction of pain and exudate in the second visit. Granulation tissue appeared in the ulcer after 1 week. The problems we noticed in this study were, increased growth of callus in plantar ulcers and difficulty to apply PRF in ulcers with small openings. This is because PRF is not a fluid substance, it is a fibrin matrix. But there is risk of recurrence of ulcer, if there is no adequate pressure relief and control of causative factors. The main limitation of the study is there is no control group, so that comparison can be made with the standard dressing technique. The small number of subjects in the study is also an important limitation.

\section{CONCLUSIONS}

- Chronic leg and foot ulcers showed significant improvement with topical autologous platelet rich fibrin therapy at the end of study period.

- $\quad$ PRF is a safe, convenient, easy-to-use therapy with significant potential for healing chronic ulcers.

- PRF therapy is cost effective, painless and can be done as an outpatient procedure.

- $\quad$ PRF treatment is a promising alternative to advanced treatment strategies, if standard therapy fails.

Data sharing statement provided by the authors is available with the full text of this article at jemds.com.

Financial or other competing interests: None.

Disclosure forms provided by the authors are available with the full text of this article at jemds.com. 


\section{REFERENCES}

[1] Järbrink K, Ni G, Sönnergren H, et al. Prevalence and incidence of chronic wounds and related complications: a protocol for a systematic review. Syst Rev 2016;5(1):152.

[2] Guo S, Dipietro LA. Factors affecting wound healing. J Dent Res 2010;89(3):219-29.

[3] Gottrup F, Apelqvist J, Price P, et al. Outcomes in controlled and comparative studies on non-healing wounds: recommendations to improve the quality of evidence in wound management. J Wound Care 2010;19(6):237-68.

[4] Damir A. Recent advances in management of chronic non healing diabetic foot ulcers. JIMSA 2011;24(4):21923.

[5] Dohan DM, Choukroun J, Diss A, et al. Platelet-rich fibrin (PRF): a second-generation platelet concentrate. Part I: technological concepts and evolution. Oral Surg Oral Med Oral Pathol Oral Radiol Endod 2006;101(3):e37-44.

[6] Madhavi G, Reddy IC, Arunakumari Y, et al. Clinicoetiological study of efficacy of autologous platelet rich fibrin in chronic non healing ulcers. IOSR Journal of Dental and Medical Sciences (IOSR-JDMS) 2019;18(2):51-8.

[7] Somani A, Rai R. Comparison of efficacy of autologous platelet rich fibrin versus saline dressing in chronic venous leg ulcers: a randomised controlled trial. J Cutan Aesthet Surg 2017;10(1):8-12.

[8] O'connell SM, Impeduglia T, Hessler K, et al. Autologous platelet-rich fibrin matrix as cell therapy in the healing of chronic lower-extremity ulcers. Wound Repair Regen 2008;16(6):749-56.

[9] Pinto NR, Ubilla M, Zamora Y, et al. Leucocyte- and platelet-rich fibrin (L-PRF) as a regenerative medicine strategy for the treatment of refractory leg ulcers: a prospective cohort study. Platelets 2018;29(5):468-75.

[10] Pravin AJS, Sridhar V, Srinivasan BN. Autologous platelet rich plasma (PRP) versus leucocyteplatelet rich fibrin (1PRF) in chronic non-healing leg ulcers - a randomised, open labelled, comparative study. J Evolution Med Dent Sci 2016;5(102):7460-2. 